\title{
MRI Changes After Platelet Rich Plasma Injection in Knee Osteoarthritis (Randomized Clinical Trial)
}

This article was published in the following Dove Press journal:

Journal of Pain Research

\section{Seyed Ahmad Raeissadat' Elham Ghorbani ${ }^{2}$ \\ Morteza Sanei Taheri ${ }^{3}$ \\ Reza Soleimani (D) $^{3}$ \\ Seyed Mansoor Rayegani $\mathbb{D D}^{2}$ Marzieh Babaee ${ }^{2}$ \\ Sara Payami (iD) \\ 'Physical Medicine and Rehabilitation Department, Shahid Modarres Hospital, Shahid Beheshti University of Medical Sciences, Tehran, Iran; ${ }^{2}$ Physical Medicine and Rehabilitation Research Center. Shohada-e-Tajrish Hospital, Shahid Beheshti University of Medical Sciences, Tehran, Iran; ${ }^{3}$ Radiology Department, Shohada-e-Tajrish Hospital, Shahid Beheshti University of Medical Sciences, Tehran, Iran; ${ }^{4}$ Department of Emergency Medicine, Amir al momenin Hospital, Tehran Azad University of Medical Sciences, Tehran, Iran}

Correspondence: Marzieh Babaee Physical Medicine and Rehabilitation Research Center, Shohada-e-Tajrish Hospital, Tajrish Sq, Tehran 1989934|48, Iran

$\mathrm{Tel} / \mathrm{Fax}+98212273 \quad 1 / 12$

Email rambabaee@yahoo.com
Purpose: Few papers have studied the objective effects of PRP on cartilage. In this study, we investigated the effect of PRP on cartilage characteristics by special MRI sequencing in knee osteoarthritis (IRCT registration number: 2014020413442N6).

Patients and methods: In this double blind randomized clinical trial, patients with bilateral knees osteoarthritis-grade 1, 2, and 3 were included in the study. Each patient's knees were randomly allocated to either control or treatment groups. PRP was injected in two sessions with 4 week intervals in PRP group. The VAS (visual analog scale) and WOMAC (Western Ontario and McMaster Universities Arthritis Index) were utilized and MRI was performed for all patients, before, and 8 months after treatment. The MRI sequences taken were transverse 3D TRUFISP and coronal and sagittal fat saturated proton-density. Imaging was scored according to four cartilage characteristics.

Results: 46 knees (from 23 patients) were included in this study. 23 knees in the case group and 23 knees in control group were studied. All patients were female with mean age of $57.57 \pm 5.9$ years. Mean total WOMAC and VAS changes before and after treatment in control group were $11.61 \pm 8.5$ and 1.3 \pm 1.1 respectively. In PRP group, mean total WOMAC and VAS changes showed better improvement with $20 \pm 12.3$ and $3.2 \pm 1.6$ respectively (P-value $<0.05$ ). In PRP group, all of the radiologic variables (patellofemoral cartilage volume, synovitis and medial and lateral meniscal disintegrity), with the exception of subarticular bone marrow abnormality, had significant improvement (P-value $<0.05$ ). In a comparison between the two groups, patellofemoral cartilage volume and synovitis had significantly changed in the PRP group ( $\mathrm{P}$-value $<0.05$ ).

Conclusion: In this study, in addition to the effect of PRP on VAS and WOMAC, there was a significant effect on radiologic characteristics (patellofemoral cartilage volume and synovitis). For further evaluation, a longer study with a larger sample size is recommended. Keywords: osteoarthritis, platelet rich plasma, PRP, magnetic resonance imaging, MRI, cartilage, knee

\section{Introduction}

Knee osteoarthritis is a progressive disease, which in addition to involving the tibiofemoral and patellofemoral joints, also affects their adjacent soft tissues. This disease is among the main causes of pain and disability which not only reduces the quality of life, but also imposes a financial burden on the individual as well as the healthcare system. The disease prevalence varies among different demographics: $19.3 \%$ among Iranian rural populations, and 2.8\% among Filipinos. In a study by Framingham, this prevalence was estimated to be 19\% among 50-60 year olds. ${ }^{1}$ Despite the high number of cases, a definite non-surgical cure has not been found yet. Hyaluronic acid, ozone, laser, botulinum toxin A, and other medical treatments have 
been suggested. ${ }^{2-6}$ One of the more recent modalities currently gaining attention is the various growth factor products. These include platelet rich plasma and plasma rich in growth factors; which because of having more platelet content compared to standard plasma, contains more growth factors. ${ }^{7}$ The growth factors stored in the alpha granules of the platelets play an important role in biological processes. The preparation of these products using a laboratory centrifuge is feasible and their administration is among the safest, most minimally invasive procedures. ${ }^{8,9}$

Various studies have shown the effectiveness of these products on the symptoms and functioning of patients with knee osteoarthritis. However, a number of studies have shown controversial results. ${ }^{10-12}$ One reason for these differences in results could be the study not being performed in a double-blinded manner, which could adversely affect the results. Most studies focus on the role that these products have on the subjective experiences of the patient regarding clinical symptoms, performance, and quality of life. Based on our research, a small number of studies have shifted their attention toward objective changes in the articular cartilage of the knee and its surrounding tissues. ${ }^{13}$ Special imaging methods for measuring cartilage have recently been employed in clinical management as well as in research. MRI is the most important noninvasive investigation method in the assessment of traumatic or degenerative lesions of the cartilage before and after performing treatments in the knee. This imaging method can show the structure of the joint in three dimensions, which can accurately and reliably measure the volume of cartilage, because of high soft tissue contrast. ${ }^{14-16}$ In some studies, MRI imaging has been utilized to evaluate intra-articular injections including viscosupplements, growth factors, stem cells etc., in which all except PRP (in a few controversial studies) have shown a positive effect on cartilage structure. ${ }^{17-20}$ In this study, we have therefore attempted to objectively assess the effect of PRP on knee osteoarthritis using the changes of cartilage in MRI sequences.

\section{Materials and Methods}

This study was a randomized, double blind clinical trial, performed between 2016-2017 in Shohoda-e-Tajrish and Shahid Modarres hospitals in Tehran. Patients with osteoarthritis visiting the physical and rehabilitation medicine clinics of these two centers were included in the study based on the following inclusion and exclusion criteria.

Inclusion criteria: history of pain for the last 3 months according to the ACR criteria for knee osteoarthritis along with radiologic evidence of joint destruction based on Kellgran-Lawrence criteria (including grades 1, 2, and 3).

Exclusion criteria: 1) any contraindication for performing an MRI including aneurism clips, pacemakers, non MRI-compatible metallic devices in the body and claustrophobia; 2) any form of knee injection such as viscosupplements, ozone etc. in the preceding 3 months; 3) having arthroscopic or open surgery in the past 6 months; 4) having immunodeficiency, autoimmune disease, collagen vascular disease, or diabetes; 5) history of cancer; 6) infection or inflamed lesion in the knee; 7) platelet disorder or disease; 8) use of anticoagulant or anti-platelet medication 10 days before injection; 9) use of NSAIDs 2 days before injection; 10) corticosteroid knee injection 3 weeks before injection or use of systemic corticosteroids 2 weeks before the injection; 11) hemoglobin level less than $12 \mathrm{~g} / \mathrm{dL}$ and platelets less than 150,000/microliter; 12) history of severe knee trauma; 13) age of less than 45 and higher than 65 ; 14) history of vasovagal shock; 15) pregnancy and lactation; 16) genu valgum or genu varum more than 20 degrees.

After patient selection, the aims, methods, current scientific evidence, benefits and possible risks of the study were described to the patients by a physical medicine and rehabilitation doctor. After this stage, all participants who had signed the informed consent form were included in the study. The form and trial were approved by the ethics committee of Shahid Beheshti University of Medical Sciences (IRCT registration number: 2014020413442N6), this was conducted in accordance with the Declaration of Helsinki. After the patients entered the study, personal information such as age, gender, and grade of osteoarthritis (Kellgren-Lawrence Grading scale in plain radiograph) was recorded. Afterwards, for each patient, a VAS (Visual Analog Scale) for pain assessment as well as a WOMAC arthritis index (Western Ontario and McMaster Universities) for functional assessment were completed with the help of a physiatrist resident. The VAS is a psychometric tool, which is used to document the severity of symptoms of a person's illness. This tool consists of a $100 \mathrm{~mm}$ ruler, which has descriptive drawings of severity on the two sides of the ruler. The patient marks his/her symptom severity based on the descriptions. The higher the number, the worse the symptoms. The WOMAC questionnaire is one of the questionnaires used in evaluating the function of patients with rheumatologic disease; in particular knee osteoarthritis. It covers the different fields of stiffness, pain, and function in certain activities; where a higher score indicates worse function. $^{21}$ 
Knee MRI images of patients were taken by a Siemens AVANTO (1.5 Tesla) MR system using a circumferential knee coil before (10-14 days before starting the treatment) and 8 months after treatment. The sequences taken were transverse 3D TRUFISP and coronal and sagittal fat saturated protondensity (PD). All images were reviewed by a radiologist and cross-checked again in Shohada-e-Tajrish's radiology department. They were unaware of the case and control groups or whether the images were taken before or after treatment. All MR images were evaluated in an unpaired manner. Four different characteristics of the joint were scored in the following manner.

1) Volume of patellofemoral cartilage: the patellofemoral cartilage was evaluated using 3D slicer software (a free platform for viewing and analysis of images in a software specific manner). All axial images were manually segmented in TRUFISP 3D sequence so that the cartilage area was separated from the nearby bone and soft tissue. Afterwards, the volume of the cartilage was calculated by multiplying the sum of the cartilage area of all the images by the thickness of the slide using 3D slicer $^{22}$ (Figure 1).

2) Subarticular bone marrow abnormality: subarticular bone marrow abnormality was scored in 15 different areas using the WORMS score. This was defined by increases in signal intensity in normal fat containing epiphyseal bone marrow in fat-saturated PD sequence. Each of these 15 regions were scored from 0 to 3 based on the level of abnormality; 0 : no signal increase, 1: less than or equal to $25 \%$ of the area, $2: 25-50 \%$ of the area, $3:$ more or equal to $50 \%$. $^{22}$

3) Internal and external meniscal integrity: the anterior and posterior horns and body of the medial and lateral menisci were separately evaluated and scored from 0-3 based on sagittal and coronal images; 0: intact, 1: globular signal inside the meniscus which is not adjacent to the articular surface, 2: linear signal inside the meniscus which is not adjacent to the articular surface, 3: abnormal signal inside the meniscus which has reached the articular surface. $^{23}$

4) Synovitis: the existence of signs of inflammation in the synovium was scored as 0 or $1 .^{22}$

The patients' knees were randomly (based on a random table of numbers) divided into two groups. Both knees had exercise therapy prescribed to them, while the study knee also had a PRP injection (group A: PRP and exercise; group B: exercise alone, Figure 2). In both knees, exercise was taught by a physiatrist. The exercise protocol employed was multi-angle isometric exercise of muscles around the knee (quadriceps, thigh abductor and adductors) as well as stretching of the hamstrings 3 times a day, 10 times for each move and 10 seconds each time. After 4 weeks, strengthening exercises for the quadriceps, adductors, and abductors were taught to the patients.

PRP was prepared using a Royagen kit. In order not to prolong the content, the details of the PRP protocol are similar to those of our previous papers. ${ }^{24-26}$ PRP preparations in this study contained leukocytes (LR-PRP) and platelet concentrations were 4-6 times normal. In the PRP group, $500 \mathrm{mg}$ of paracetamol without codeine could be ingested for pain relief after injection. In case of further pain, paracetamol with codeine was prescribed as needed and patients were forbidden from using any forms of NSAIDs or steroids. The patients could restart all daily activities one week post injection. In the study group, exercise therapy commenced one week after injection, which started with lower intensity and increased gradually. In the control group, exercise therapy started immediately and the patients in this group were also asked to use $500 \mathrm{mg}$ of paracetamol without codeine in case of pain; and if the pain was not controlled, paracetamol with codeine was to be used. The second injection was performed 28 days (4 weeks) after the first.

The prescribed medications and the importance of adherence to the prescribed drugs as well as refraining from use of drugs which affect platelet activity were explained. The patients were asked to contact the researchers in case of any problems.

All patients were followed up 32 weeks after therapy, when the VAS and WOMAC forms were completed again for them and another MRI was performed.

The final data were entered into SPSS-16 for analysis.

Normality of the variables' distribution was assessed using the Shapiro Wilk test. Comparisons for before and after each intervention were performed separately for each group using $t$-test, paired $t$-test, and ANOVA for variables with normal distribution. For non-parametric variables, Wilcoxon signed rank, Mann-Whitney and Kruskal Wallis tests were employed. Qualitative data were expressed using value and percentage. In order to assess the relation between quantitative variables, Pearson's correlation coefficient and Spearman's coefficient were utilized.

\section{Results}

46 knees (of 23 patients) were entered into this study, 23 of which entered the control group and 23 of which were in the intervention group. 2 knees from each group were 

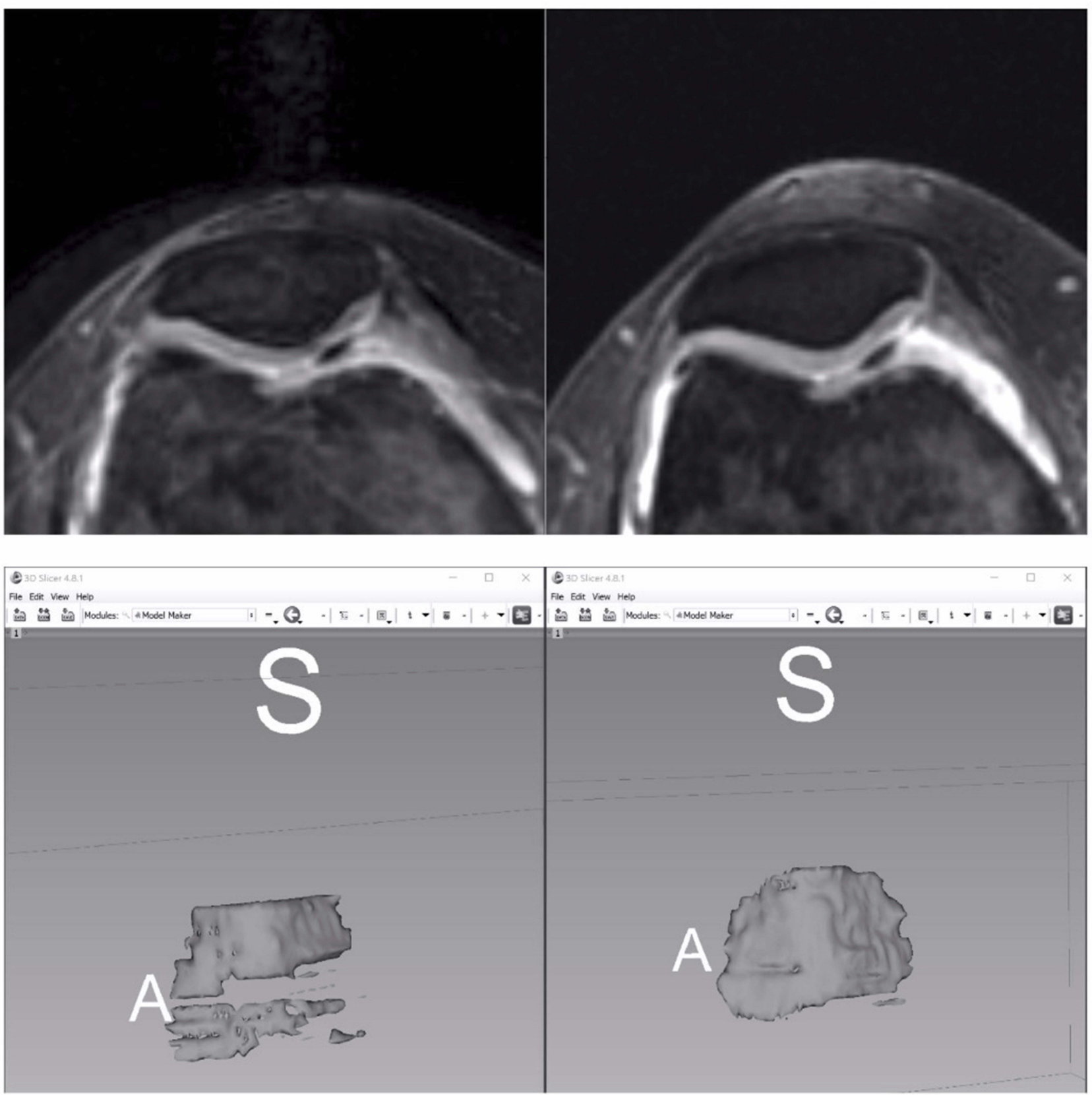

Figure I The patellofemoral cartilage was evaluated using 3D slicer software (a free platform for viewing and analysis of images in a software specific manner). Left picture before PRP injection and right picture after PRP injection.

eliminated because of failure to show up in follow up, leaving 21 knees in each group after 8 months (Figure 2). All patients were female, with an average age of $57.57 \pm 5.9$ years and average BMI of $28.49 \pm 3.24 \mathrm{Kg} / \mathrm{m}^{2}$.

Except for VAS score, none of the characteristics had any significant difference between groups (Table 1). The average VAS showed a significant improvement inter-group and between groups $(\mathrm{P}<0.05)$ (Table 2).
In addition, there was a meaningful difference between the before and after treatment WOMAC scores of both groups. There was also a difference between the after treatment WOMAC scores of both groups except for the pain subgroup which had borderline P-Value (Table 2).

In the PRP group, patellofemoral cartilage volume, synovitis, and medial and lateral meniscal disintegrity showed significant improvement after treatment. In the 


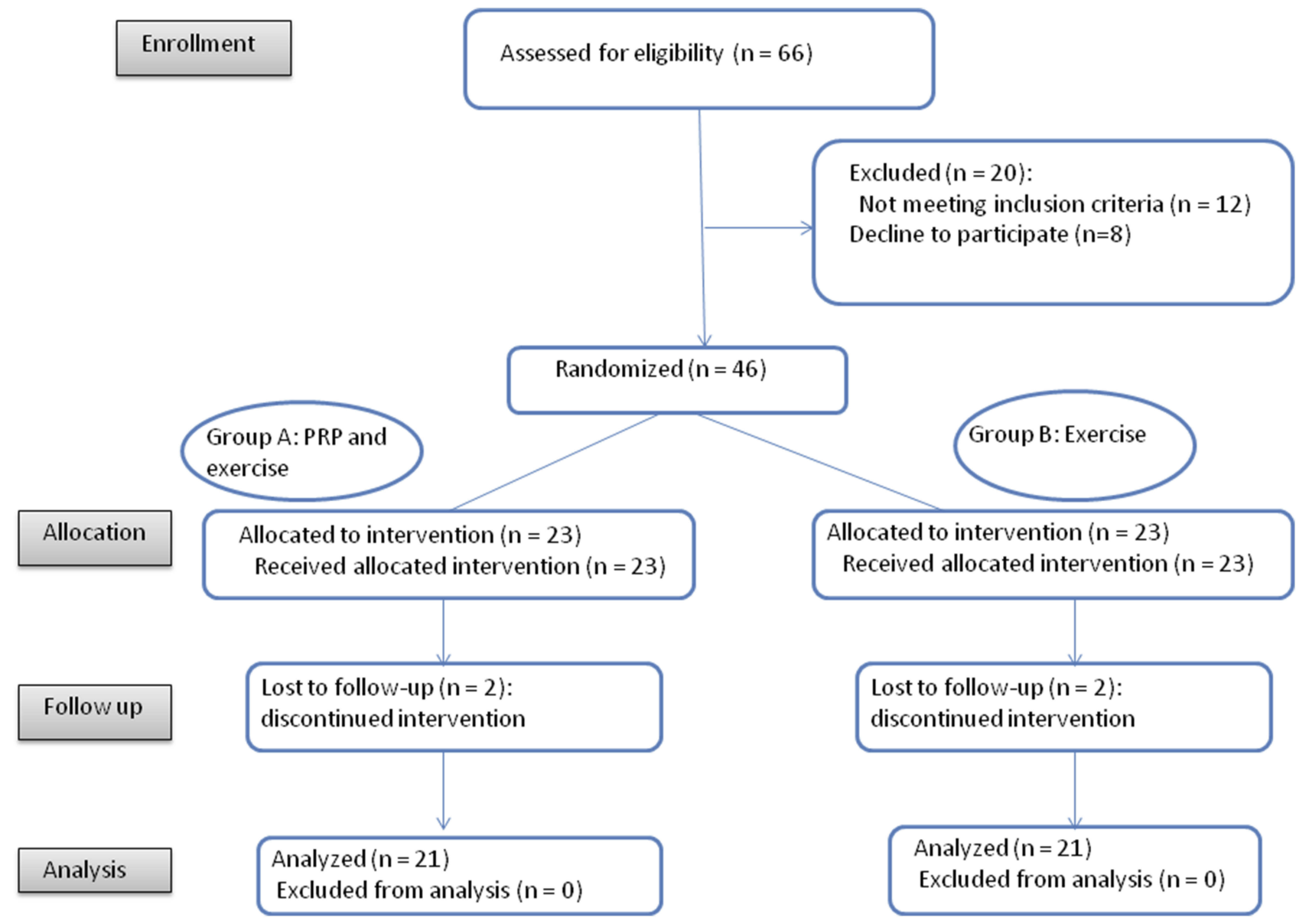

Figure 2 CONSORT flow diagram.

control group, only patellofemoral cartilage volume showed meaningful improvement (Table 3).

Table I Baseline Characteristics of Study Population

\begin{tabular}{|c|c|c|c|}
\hline & Control & PRP & P-value \\
\hline Kellgren Lawrence grade, number (\%) & & & \\
\hline 1 & 26.3 & 26.3 & 1 \\
\hline 2 & 52.6 & 52.6 & 1 \\
\hline 3 & 21.1 & 21.1 & 1 \\
\hline Total WOMAC (Mean \pm SD) & $28 \pm 11$ & $35 \pm 16$ & 0.129 \\
\hline Pain & $6.47 \pm 3.09$ & $8.14 \pm 4.56$ & 0.175 \\
\hline Stiffness & $1.6 \pm 1.4$ & $2.5 \pm 2.2$ & 0.134 \\
\hline Difficulty & $20.19 \pm 8.2$ & $24.28 \pm 10.95$ & 0.180 \\
\hline VAS (Mean \pm SD) & $4.7 \pm 1.84$ & $6 \pm 2.07$ & 0.047 \\
\hline Patellofemoral cartilage volume & 1012.68 & 1041.47 & 0.1 \\
\hline (Mean \pm SD) & \pm 259.24 & \pm 323.01 & \\
\hline $\begin{array}{l}\text { Subarticular bone marrow } \\
\text { abnormality (Mean } \pm \mathrm{SD} \text { ) }\end{array}$ & $1.83 \pm 1.69$ & $2.16 \pm 2$ & 0.333 \\
\hline $\begin{array}{l}\text { Medial and lateral meniscal } \\
\text { disintegrity (Mean } \pm \text { SD) }\end{array}$ & $2.27 \pm 2.7$ & $2.44 \pm 2.7$ & 0.166 \\
\hline Synovitis (\%) & 61.9 & 66.7 & 0.055 \\
\hline
\end{tabular}

In comparison of the two groups, improvement in patellofemoral cartilage volume and synovitis was better in the PRP group (Table 3).

Because of the low number of patients, no relation was found between the radiologic parameters and grade of osteoarthritis.

\section{Discussion}

This clinical trial showed the positive effects of PRP on MRI findings. In our study MRI changes including patellofemoral cartilage volume and synovitis were better in the PRP group.

At present, systematic reviews and meta analyses have shown the positive role of PRP in pain reduction and functional improvement in patients with mild to moderate osteoarthritis. The findings of these studies have been obtained in a subjective manner. ${ }^{10,12,27}$ Few studies have compared osteoarthritis treatments such as hyaluronic acid, growth factors, stem cells etc., objectively; using MR imaging. ${ }^{28-30}$ Furthermore, to the best of our knowledge, few studies have assessed the effect of PRP on radiologic and MRI findings. ${ }^{19,31,32}$ 
Table 2 Mean \pm SD of WOMAC and VAS After 8 Months Treatment in Both Groups

\begin{tabular}{|c|c|c|c|c|}
\hline WOMAC Parameters & Group & 8 Months & $\begin{array}{l}\text { p-value }{ }^{a} \\
\text { (Inter Group) }\end{array}$ & $\begin{array}{l}\text { p-value }{ }^{a} \\
\text { (Between Groups) }\end{array}$ \\
\hline Pain & $\begin{array}{l}\text { PRP } \\
\text { Control }\end{array}$ & $\begin{array}{l}3.85 \pm 3.4 \\
4.04 \pm 2.45\end{array}$ & $\begin{array}{l}0.001 \\
0.001\end{array}$ & 0.056 \\
\hline Stiffness & $\begin{array}{l}\text { PRP } \\
\text { Control }\end{array}$ & $\begin{array}{l}0.76 \pm 0.88 \\
I \pm I . I\end{array}$ & $\begin{array}{l}0.001 \\
0.036\end{array}$ & 0.036 \\
\hline Functional capacity & $\begin{array}{l}\text { PRP } \\
\text { Control }\end{array}$ & $\begin{array}{l}10.15 \pm 8.3 \\
12.05 \pm 6.7\end{array}$ & $\begin{array}{l}0.001 \\
0.001\end{array}$ & 0.038 \\
\hline VAS & $\begin{array}{l}\text { PRP } \\
\text { Control }\end{array}$ & $\begin{array}{l}2.76 \pm 2.07 \\
3.42 \pm 1.83\end{array}$ & $\begin{array}{l}0.001 \\
0.001\end{array}$ & 0.001 \\
\hline
\end{tabular}

Note: ${ }^{a}$ P-Value $<0.05$ was considered significant.

Table 3 Radiologic Parameters After Treatment in Both Groups

\begin{tabular}{|c|c|c|c|c|}
\hline Radiologic Parameters & Group & 8 Months & $\begin{array}{l}\text { p-value }{ }^{a} \\
\text { (Inside Group) }\end{array}$ & $\begin{array}{l}\text { p-value }{ }^{a} \\
\text { (Between Groups) }\end{array}$ \\
\hline Patellofemoral cartilage volume (Mean \pm SD) & $\begin{array}{l}\text { PRP } \\
\text { Control }\end{array}$ & $\begin{array}{l}1336.88 \pm 295.83 \\
\mid 105.1 \pm 262.62\end{array}$ & $\begin{array}{l}0.001 \\
0.05\end{array}$ & 0.001 \\
\hline Subarticular bone marrow abnormality (Mean \pm SD) & $\begin{array}{l}\text { PRP } \\
\text { Control }\end{array}$ & $\begin{array}{l}1.6 \pm 1.9 \\
1.5 \pm 1.5\end{array}$ & $\begin{array}{l}0.142 \\
0.058\end{array}$ & 0.6 \\
\hline Medial and lateral meniscal disintegrity (Mean \pm SD) & $\begin{array}{l}\text { PRP } \\
\text { Control }\end{array}$ & $\begin{array}{l}1.6 \pm 2.1 \\
1.5 \pm 2.1\end{array}$ & $\begin{array}{l}0.01 \\
0.196\end{array}$ & 0.8 \\
\hline Synovitis (\%) & $\begin{array}{l}\text { PRP } \\
\text { Control }\end{array}$ & $\begin{array}{l}28.5 \\
52.3\end{array}$ & $\begin{array}{l}0.008 \\
0.5\end{array}$ & 0.026 \\
\hline
\end{tabular}

Note: ${ }^{\text {a }}$-Value $<0.05$ was considered significant.

In 2013, Halpern et al studied the effect of PRP on clinical signs and MRI findings in knees of 15 patients suffering from osteoarthritis. $6 \mathrm{~mL}$ of PRP prepared from $20 \mathrm{~mL}$ of patient blood was injected. Clinical signs were assessed using WOMAC and VAS, while MRIs were performed via a 1.5 Tesla scanner and fast spin echo images in 3 planes; and afterwards reported by 2 radiologists. The patients were followed after 1 week as well as 1, 3, 6, and 12 months. After 6 and 12 months, the WOMAC score and VAS improved. From the 15 knees with MRI images, 12 (80\%) did not show changes toward exacerbation of osteoarthritis in the patellofemoral joint. Furthermore, in 12 knees $(80 \%)$, during one year no change was seen in the radiologic appearance of the lateral tibiofemoral joint. Similarly, no changes were reported in the involvements of the medial compartment in $73.3 \%$ of cases; although this did not have statistical significance. ${ }^{19}$ The radiologic appearance of one knee showed improvement after 1 year. In the end, the authors expressed that some studies had shown $4-6 \%$ yearly reduction in cartilage volume in an osteoarthritic compartment, which was in contrast with their current study results regarding PRP treatment. Moreover, it is possible that one injection of PRP can reduce cartilage loss. In comparison, in our study, patellofemoral cartilage volume and synovitis improved further in the PRP group. Although Halpern's study did not show changes in the thickness of cartilage, it did not show any loss of cartilage either. These findings could be related to the number of injections which was double in our study and single injection in Halpern's study.

In 2018, Buendía-López studied the effect of PRP, hyaluronic acid, and NSAIDS on MRI findings. 98 patients (33 in the NSAID group, 32 in hyaluronic acid group, and 33 in the PRP group) completed the study. In the LP-PRP group (leukocyte poor PRP), an injection was performed once and the patients of all three groups were assessed with WOMAC and VAS 26 and 52 weeks into follow up. In addition, an X-ray and MRI were performed at the beginning and end (52 weeks) of the study. ${ }^{31}$ In WOMAC and VAS-pain, the results of the LP-PRP group were better. In this study the change of grade of osteoarthritis from grade 
1 to 2 was $17 \%$, while grade 2 did not show significant change. No increase in thickness of cartilage was observed; although no meaningful decrease was observed between the three groups either. This study was without a placebo group and a single LP-PRP injection with a concentration of 3.8 times was assessed in only grade 1 and 2. In Hart's study, which observed the effect of 9 PRP injections in one year on MRI findings, no regeneration of cartilage was seen. Patients with grade 2 and 3 osteoarthritis were entered into this study and the PRP had 2 to 2.5 times concentration. ${ }^{32}$ These differences in results of various studies could be due to the presence of leukocytes in some PRP preparations, PRP concentration, number of PRP injections, follow up time, and grade of osteoarthritis.

In 2018, in addition to clinical findings, Ahmad assessed the changes in cartilage using sonography in 2 groups of PRP and hyaluronic acid injections. In both groups, 3 injections were performed with a 2 week intervals between injections. In both groups, after 3 and 6 months of follow up, clinical improvement was observed; with the PRP group showing better improvement than the hyaluronic acid group. The improvements in the PRP group showed less effusion, reduced synovial hypertrophy, and improved vascularity scores in ultrasound assessment. ${ }^{33}$ Although the imaging method is different from our study, the radiologic changes bear similarities with our study's results.

In 2016, Smara also studied a platelet-derived product named autologous platelet lysates and its effects on cartilage in MRIs. 15 patients with grade 1 and 2 osteoarthritis had this product injected 2 times, every 3 weeks. After one year follow up, the thickness of cartilage on both the tibial and femoral sides of the joint showed significant improvement. ${ }^{20}$

What is evident is that MRI imaging plays an important role in faster assessment of patients with osteoarthritis. In Framingham's study, it was shown that patients who lack any signs of knee osteoarthritis in plain radiographs could have some MRI changes indicative of the beginning of osteoarthritis. ${ }^{34}$ Therefore, MRI is one of the most sensitive tools for evaluation of changes in osteoarthritis and its response to treatment. Use of MRI in order to assess the effect of treatment, in particular the somewhat newer modalities, can determine their actual effects. In this study, we tried to observe the visible effects of PRP. The presence of a control group, the design of the study as a clinical trial, and being among the first studies using MRI imaging to assess PRP treatment are the positive aspects of our study. The relatively small number of subjects, shorter follow up time, assessment of mild to moderate grades of osteoarthritis, results being affected by other variables such as a high BMI as well as lack of comparison with other treatment methods can be among the shortcomings of this study. We attempted, in this research, to study the effects of PRP, as one of the more commonly accepted treatments for mild to moderate osteoarthritis, on MRI findings.

\section{Conclusion}

It was observed that compared to the control group, PRP was effective in the improvement of patellofemoral cartilage volume and synovitis. Even though, a longer study with a higher number of subjects and comparison with other treatment methods is recommended for further evaluation.

\section{Data Sharing Statement}

The authors intend to share individual participant data that underline the results reported in this article after deidentification (text, tables, figures, and appendix). Study protocol, statistical analysis plane, informed consent form and clinical study report will be made available and the data will be accessible by email to rambabaee@yahoo.com. To gain access, data requestors will need to sign a data access agreement. Immediately following publication and ending 2 years following article publication they will be made available.

\section{Acknowledgments}

We thank Ms. Mahshid Zaman for her assistance with collecting the patients' images and data.

An abstract of this paper titled "Volumetric findings of MRI after platelet rich plasma injection in knee osteoarthritis (A randomized clinical trial)" was presented at the 12th International Society of Physical and Rehabilitation Medicine Conference, as a conference talk with interim findings; and was published in "Poster Abstracts" in the Annals of Physical and Rehabilitation Medicine Journal (https://doi.org/10.1016/j.rehab.2018.05.022); ${ }^{35}$ and was also presented as an oral presentation at the Annual European Congress of Rheumatology EULAR 2018. ${ }^{36}$

\section{Disclosure}

The authors report no conflicts of interest in this work.

\section{References}

1. Felson DT The epidemiology of knee osteoarthritis: results from the Framingham Osteoarthritis Study. Paper presented at: Seminars in arthritis and rheumatism; 1990. doi:10.1099/00221287-136-2-327 
2. Kon E, Filardo G, Drobnic M, et al. Non-surgical management of early knee osteoarthritis. Knee Surg Sports Traumatol Arthroscopy. 2012;20(3):436-449. doi:10.1007/s00167-011-1713-8

3. Raeissadat SA, Tabibian E, Rayegani SM, Rahimi-Dehgolan S, BabaeiGhazani A. An inestigation into the efficacy of intra-articular ozone (O2-O3) injection in patients with knee osteoarthritis: a systematic review and meta-analysis. J Pain Res. 2018;11:2537-2550. doi:10.2147/JPR. S175441

4. Raeissadat SA, Rayegani SM, Forogh B, Abadi PH, Moridnia M, Dehgolan SR. Intra-articular ozone or hyaluronic acid injection: which one is superior in patients with knee osteoarthritis? A 6-month randomized clinical trial. J Pain Res. 2018;11:111. doi: $10.2147 /$ JPR.S142755

5. Rayegani SM, Raeissadat SA, Heidari S, Moradi-Joo M. Safety and effectiveness of low-level laser therapy in patients with knee osteoarthritis: a systematic review and meta-analysis. J Lasers Med Sci. 2017;8(Suppl 1):S12. doi:10.15171/jlms.2017.s3

6. Raeissadat SA, Rayegani SM, Sedighipour L, et al. The efficacy of electromyographic biofeedback on pain, function, and maximal thickness of vastus medialis oblique muscle in patients with knee osteoarthritis: a randomized clinical trial. J Pain Res. 2018;11:2781. doi:10.2147/JPR.S169613

7. Raeissadat SA, Rayegani SM, Ahangar AG, Abadi PH, Mojgani P, Ahangar OG. Efficacy of intra-articular injection of a newly developed Plasma Rich in Growth Factor (PRGF) versus hyaluronic acid on pain and function of patients with knee osteoarthritis: a single-blinded randomized clinical trial. Clin Med Insights. 2017;10:1179544117733452.

8. Raeissadat SA, Babaee M, Hashemi Z. Application of platelet-rich plasma and other platelet-derivative products in Iranian studies. koomesh. 2019;21(3):408-422.

9. Raeissadat SA, Babaee M, Rayegani SM, et al. An overview of platelet products (PRP, PRGF, PRF, etc.) in the Iranian studies. Future Sci OA. 2017;3(4):FSO231. doi:10.4155/fsoa-2017-0045

10. Laudy AB, Bakker EW, Rekers M, Moen MH. Efficacy of platelet-rich plasma injections in osteoarthritis of the knee: a systematic review and meta-analysis. Br J Sports Med. 2015;49 (10):657-672. doi:10.1136/bjsports-2014-094036

11. Campbell KA, Saltzman BM, Mascarenhas R, et al. Does intra-articular platelet-rich plasma injection provide clinically superior outcomes compared with other therapies in the treatment of knee osteoarthritis? A systematic review of overlapping meta-analyses. Arthroscopy. 2015;31(11):2213-2221. doi:10.1016/j.arthro.2015.03.041

12. Khoshbin A, Leroux $T$, Wasserstein D, et al. The efficacy of platelet-rich plasma in the treatment of symptomatic knee osteoarthritis: a systematic review with quantitative synthesis. Arthroscopy. 2013;29(12):2037-2048. doi:10.1016/j.arthro.2013.09.006

13. Meheux CJ, McCulloch PC, Lintner DM, Varner KE, Harris JD. Efficacy of intra-articular platelet-rich plasma injections in knee osteoarthritis: a systematic review. Arthroscopy. 2016;32(3):495-505. doi:10.1016/j.arthro.2015.08.005

14. Gold GE, Chen CA, Koo S, Hargreaves BA, Bangerter NK. Recent advances in MRI of articular cartilage. Am J Roentgenol. 2009;193 (3):628-638. doi:10.2214/AJR.09.3042

15. Hill CL, Hunter DJ, Niu J, et al. Synovitis detected on magnetic resonance imaging and its relation to pain and cartilage loss in knee osteoarthritis. Ann Rheum Dis. 2007;66(12):1599-1603. doi:10.1136/ ard.2006.067470

16. Hunter DJ, Li J, LaValley M, et al. Cartilage markers and their association with cartilage loss on magnetic resonance imaging in knee osteoarthritis: the Boston osteoarthritis knee study. Arthritis Res Ther. 2007;9(5):R108. doi:10.1186/ar2314

17. Cubukcu D, Ardic F, Karabulut N, Topuz O. Hylan G-F 20 efficacy on articular cartilage quality in patients with knee osteoarthritis: clinical and MRI assessment. Clin Rheumatol. 2005;24(4):336-341. doi:10.1007/s10067-004-1043-z
18. Durmus D, Alayli G, Bayrak IK, Canturk F. Assessment of the effect of glucosamine sulfate and exercise on knee cartilage using magnetic resonance imaging in patients with knee osteoarthritis: a randomized controlled clinical trial. J Back Musculoskelet Rehabil. 2012;25 (4):275-284. doi:10.3233/BMR-2012-0336

19. Halpern B, Chaudhury S, Rodeo SA, et al. Clinical and MRI outcomes after platelet-rich plasma treatment for knee osteoarthritis. Clin J Sport Med. 2013;23(3):238-239. doi:10.1097/JSM.0b013e31827c3846

20. Samara O, Al-Ajlouni J, Al-Najar M, et al. Intra-articular autologous platelet lysates produce positive MRI structural changes in early and intermediate knee osteoarthrosis. PJR. 2016;27:1.

21. Gandek B. Measurement properties of the Western Ontario and McMaster Universities osteoarthritis index: a systematic review. Arthritis Care Res (Hoboken). 2015;67(2):216-229. doi:10.1002/acr.v67.2

22. Peterfy C, Guermazi A, Zaim S, et al. Whole-organ magnetic resonance imaging score (WORMS) of the knee in osteoarthritis. Osteoarthritis Cartilage. 2004;12(3):177-190. doi:10.1016/j.joca. 2003.11.003

23. Stoller DW, Martin C, Crues J 3rd, Kaplan L, Mink JH. Meniscal tears: pathologic correlation with MR imaging. Radiology. 1987;163 (3):731-735. doi:10.1148/radiology.163.3.3575724

24. Raeissadat SA, Rayegani SM, Babaee M, Ghorbani E. The effect of platelet-rich plasma on pain, function, and quality of life of patients with knee osteoarthritis. Pain Res Treat. 2013;2013.

25. Raeissadat SA, Rayegani SM, Hassanabadi H, et al. Knee osteoarthritis injection choices: platelet-rich plasma (PRP) versus hyaluronic acid (a one-year randomized clinical trial). Clin Med Insights. 2015;8 (CMAMD):S17894.

26. Raeissadat SA, Karimzadeh A, Hashemi M, Bagherzadeh L. Safety and efficacy of platelet-rich plasma in treatment of carpal tunnel syndrome; a randomized controlled trial. BMC Musculoskelet Disord. 2018;19(1):49. doi:10.1186/s12891-018-1963-4

27. Chang K-V, Hung C-Y, Aliwarga F, Wang T-G, Han D-S, Chen W-S. Comparative effectiveness of platelet-rich plasma injections for treating knee joint cartilage degenerative pathology: a systematic review and meta-analysis. Arch Phys Med Rehabil. 2014;95(3):562-575. doi:10.1016/j.apmr.2013.11.006

28. Forney MC, Gupta A, Minas T, Winalski CS. Magnetic resonance imaging of cartilage repair procedures. Magn Reson Imaging Clin. 2014;22(4):671-701. doi:10.1016/j.mric.2014.07.008

29. Wang Y, Hall S, Hanna F, et al. Effects of Hylan GF 20 supplementation on cartilage preservation detected by magnetic resonance imaging in osteoarthritis of the knee: a two-year single-blind clinical trial. BMC Musculoskelet Disord. 2011;12(1):195. doi:10.1186/14712474-12-195

30. Woollard JD, Gil AB, Sparto P, et al. Change in knee cartilage volume in individuals completing a therapeutic exercise program for knee osteoarthritis. J Orthop Sports Phys Ther. 2011;41 (10):708-722. doi:10.2519/jospt.2011.3633

31. Buendía-López D, Medina-Quirós M, Marín MÁF-V. Clinical and radiographic comparison of a single LP-PRP injection, a single hyaluronic acid injection and daily NSAID administration with a 52-week follow-up: a randomized controlled trial. J Orthopaedics Traumatol. 2018;19(1):3. doi:10.1186/s10195-018-0501-3

32. Hart R, Safi A, Komzák M, Jajtner P, Puskeiler M, Hartová P. Platelet-rich plasma in patients with tibiofemoral cartilage degeneration. Arch Orthop Trauma Surg. 2013;133(9):1295-1301. doi:10.1007/s00402-013-1782-x

33. Ahmad HS, Farrag SE, Okasha AE, et al. Clinical outcomes are associated with changes in ultrasonographic structural appearance after platelet-rich plasma treatment for knee osteoarthritis. Int $J$ Rheum Dis. 2018;21(5):960-966. doi:10.1111/apl.2018.21.issue-5

34. Guermazi A, Niu J, Hayashi D, et al. Prevalence of abnormalities in knees detected by MRI in adults without knee osteoarthritis: population based observational study (Framingham Osteoarthritis Study). BMJ (Clinical Research Ed). 2012;345:e5339. 
35. Raeissadat S, Ghorbani E, Rayegani S, Taheri MS, Babaee M, Soleimani R. Volumetric findings of MRI after platelet rich plasma injection in knee osteoarthritis (A randomized clinical trial). Ann Phys Rehabil Med. 2018;61:e9. doi:10.1016/j.rehab.2018.05.022
36. Raeissadat S, Ghorbani E, Sanei M, Rayegani S, Babaee M, Soleimani R. AB0975 Does Platelet Rich Plasma Change the Volumetric Findings of MRI Imaging?(A Randomised Clinical Trial). BMJ Publishing Group Ltd. 2018.

\section{Publish your work in this journal}

The Journal of Pain Research is an international, peer reviewed, open access, online journal that welcomes laboratory and clinical findings in the fields of pain research and the prevention and management of pain. Original research, reviews, symposium reports, hypothesis formation and commentaries are all considered for publication. The manuscript

Submit your manuscript here: https://www.dovepress.com/journal-of-pain-research-journa management system is completely online and includes a very quick and fair peer-review system, which is all easy to use. Visit http:// www.dovepress.com/testimonials.php to read real quotes from published authors. 